\title{
Responses to Affective Stimuli in Monkeys with Entorhinal or Perirhinal Cortex Lesions
}

\author{
Martine Meunier, ${ }^{1}$ Laetitia Cirilli, ${ }^{1}$ and Jocelyne Bachevalier ${ }^{2}$ \\ ${ }^{1}$ Institut des Neurosciences Cognitives de la Méditerranée, Unité Mixte de Recherche 6193, Centre National de la Recherche Scientifique and Aix-Marseille \\ Université, 13602 Marseille, France, and ${ }^{2}$ Yerkes National Primate Center and Emory University, Atlanta, Georgia 30329
}

\begin{abstract}
Recent efforts to define the functions of the primate rhinal (entorhinal and perirhinal) cortical areas have focused on their interaction with the hippocampus in the mediation of normal memory. Less is known on the functional meaning of their strong connections to the amygdala, a key substrate for emotion. A previous study (Meunier and Bachevalier, 2002) showed evidence that complete rhinal ablations yield changes in monkeys' behavioral responses to affectively salient stimuli. Here, we studied monkeys with separate entorhinal or perirhinal ablations in the same paradigm, where responses were triggered by four stimuli: an unfamiliar human, a conspecific stimulus, a toy snake, and a familiar (generally rewarded) junk object. The two separate lesions produced similar changes, and each replicated the effects of complete rhinal lesions (i.e., attenuated affiliation and enhanced defense). Failure to modulate responses based on previous experience (i.e., memory difficulties) may explain these affective changes. This interpretation does not account, however, for the sparing of some memory-dependent modulations of defense, nor for the lack of correlation between the animals' affective changes and their own recognition memory performance. Alternatively, rhinal damage may introduce a negative bias in the risk assessment of affectively salient stimuli, a proposal more compatible with Gray and McNaughton's (2000) anxiety-centered view of medial temporal functions, than with prominent mnemonic/perceptual functional models of the hippocampal/rhinal duo. Reconciling the two perspectives may improve our understanding of rhinal functions.
\end{abstract}

Key words: social behavior; emotion; fear; anxiety; reward; hippocampus; amygdala

\section{Introduction}

The amygdala and hippocampus (CA fields, dentate gyrus, and subicular complex) form the inner core of the primate medial temporal lobe (MTL). Two long-standing streams of research, one originating in Klüver and Bucy's (1939) report of affective changes in monkeys, the other in Scoville and Milner's (1957) description of amnesic patient HM, have led to an association of these two structures with distinct functional domains, emotion for the amygdala, and memory for the hippocampus. The outer components of the MTL, in particular the rhinal (entorhinal and perirhinal) areas, are closely interconnected with both the amygdala and hippocampus (Amaral, 1992). However, because their functional importance was acknowledged in primate research, $\sim 10$ years ago, most of the effort has been dedicated to contrast rhinal cortex contribution to memory to that of the hippocampus (for a recent overview, see Murray et al., 2005 and companion papers). The possible implication of the rhinal cortex in affective functions through, for example, its dense connections with the amygdala, has received little attention in primates, despite sup-

Received May 8, 2006; revised June 15, 2006; accepted June 16, 2006.

This work was supported by Centre National de la Recherche Scientifique (Programme International de Collaboration Scientifique), the National Institute of Mental Health (NIMH-IRP and MH58846), and the National Institute of Child and Human Development (HD35471).We thank Drs. M. Mishkin and E. A. Murray for their help and support throughout this study.

Correspondence should be addressed to Jocelyne Bachevalier, Yerkes National Primate Research Center, Emory University, 954 Gatewood Road, Atlanta, GA 30329. E-mail: jbachev@emory.edu.

D0I:10.1523/JNEUROSCI.1949-06.2006

Copyright $\odot 2006$ Society for Neuroscience $\quad$ 0270-6474/06/267718-05\$15.00/0 porting experimental (Otto et al., 2000) and theoretical (Gray and McNaughton, 2000) evidence from the rat literature.

In a previous study, the behavioral responses to affectively salient stimuli of monkeys with rhinal cortex ablations (Meunier and Bachevalier, 2002) were compared with those of monkeys with neurotoxic or aspiration amygdala lesions (Meunier et al., 1999). Four stimuli probed affective functions (construed in a broad sense encompassing social behavior, emotion, and motivation): two with a social component (an unfamiliar human and a conspecific stimulus) and two nonsocial items (a generally rewarded object and a toy snake). A detailed ethogram was built to quantify behaviors during three weekly presentations of each stimulus. In this paradigm, rhinal cortex ablations yielded none of the Klüver-Bucy-like symptoms (hyperorality, hypermetamorphosis, excessive affiliation, and diminished fear) recorded after lesions involving the amygdala. Rather, they led to subtle behavioral changes that were opposite in direction, namely, they attenuated affiliative responses and heightened defensiveness. Thus, rhinal damage can interfere with responses to affectively salient stimuli in monkeys, and in a way radically different from amygdala damage. Here, we evaluated the effects of separate entorhinal or perirhinal ablations in the same behavioral paradigm to determine whether damage restricted to rhinal cortex (i.e., sparing the overlying amygdala) reliably resulted in affective changes, and if yes, which of its two components was mostly responsible for it. Because each of the separate lesions was found equally efficient to produce the changes seen after complete rhi- 
nal ablations, we then attempted to clarify the link between this affective impact and the memory abilities of the same monkeys, those expressed through the present study, as well as the object recognition abilities measured earlier using delayednonmatching-to-sample (DNMS) (Meunier et al., 1993). A preliminary report appeared previously (Meunier et al., 2001).

\section{Materials and Methods}

The study was approved by the Institutional Animal Care and Use Committee of the National Institute of Mental Health. The subjects were eight individually housed, adult rhesus monkeys (Macaca mulatta), four (two male and two female) received aspiration lesions of the entorhinal cortex (ERh), and four (three male and one female) received aspiration lesions of the perirhinal cortex (PRh). Data from these two groups were compared with those of six (male) unoperated controls (N) and five (two male and three female) with combined entorhinal and perirhinal ablations (Rh) (Meunier and Bachevalier, 2002). All operated monkeys had been previously tested using DNMS with trial-unique object (Meunier et al., 1993). Postoperative DNMS testing consisted of relearning with $10 \mathrm{~s}$ delays, followed by increasing delays $(10,30,60$, and $120 \mathrm{~s})$ and listlengths of objects $(3,5$, and 10). Surgical procedures, preoperative and postoperative care, and the location and estimated volumes of damage have been detailed in Meunier et al. (1993). Briefly, ERh (area 28) lesions averaged $75 \%$ of the area volume (range $65-89 \%$ ), with slightly asymmetrical damage in two cases. Sparing was located in the medial most part of ERh. Inadvertent damage to the posterior half of PRh ranged from 25 to $61 \%$. PRh (areas 35 and 36) lesions were symmetrical and substantial in all cases, averaging $88 \%$ of the area volume (range $76-95 \%$ ). Sparing occurred in the rostrolateral part of PRh, and unintended damage was minor in all cases. Rh lesions have also been detailed previously, the present animals corresponding to cases Rh 2-4 and 6-7 in Meunier et al. (1993, 1996), respectively. Case Rh-1 did not undergo affective testing, and atypical case $\mathrm{Rh}-5$, which showed compulsive motor stereotypies rather than exaggerated defense, is discussed in detail in Meunier and Bachevalier (2002). In all five cases, damage was symmetrical and extensive, including, on average, $70 \%$ (range $42-82 \%$ ) of ERh volume together with $86 \%$ (range $77-97 \%$ ) of PRh volume, with the same sparing as separate lesions, and only minor unintended damage. Thus, in all $\mathrm{ERh}, \mathrm{PRh}$, or Rh cases, the anterior parts of rhinal areas, the main loci of interconnection with the amygdala (Stefanacci et al., 1996), were extensively and bilaterally damaged; the polar extension of perirhinal cortex, which has the densest amygdala connections, was also involved bilaterally in ERh 1-2, PRh 1 and 3, and Rh 2 and 6-7, and unilaterally in the remaining cases.

The apparatus, stimuli, recording procedure, and scoring method were identical to those used previously (Meunier et al., 1999; Meunier and Bachevalier, 2002). Monkeys were in a Wisconsin General Testing Apparatus. Each daily session lasted 9 min. Four epochs lasting 1 min each were video-recorded. A single stimulus was presented per session during the third recorded epoch for $20 \mathrm{~s}$. The experimenter wearing a rubber face mask (and staring at the animal during the last $10 \mathrm{~s}$ ) served as an unfamiliar human. A taxidermic monkey head (thrust toward the animal after the first $10 \mathrm{~s}$ ) served as a threatening conspecific stimulus. A toy rubber snake served as a negative item [toy snakes efficiently trigger monkeys' species-typical fear of snakes (Mineka et al., 1980)]. A junk object concealing a food treat (a familiar situation for monkeys with DNMS training) served as a positive item. The object was the only stimulus to be presented four times for $20 \mathrm{~s}$ instead of once per daily session. On the fourth presentation the reward was omitted to evaluate reactions to a presumably unexpected and frustrating event. The four stimuli were each presented once a week for three consecutive weeks, in a different sequence each week to all subjects. Water and food were given ad libitum after testing completion.

A sample of sessions was analyzed by two observers, one of them being unaware of the animal's group, to calculate interobserver reliability (Pearson $r=0.95$ ). Before and after stimulus presentation, the duration of six mutually exclusive activities was measured: passive, move, locomotor stereotypies, self-directed activities, and manual and oral exploration.
During stimulus presentation, the duration of 20 nonmutually exclusive activities were grouped in five nonoverlapping composite categories: aggression (yawn, head or body lunge, cage shake, mouth threat, striking attack, biting attack), affiliation (lip smack, grimace, hindquarter presentation), defense (freezing, startle, eye or head aversion, move away), approach (move toward, touch, mouth, smell), and displacement behaviors (manipulate cage or apparatus, locomotor stereotypies, and self-directed activities). This ethogram is the same as that used previously (Meunier et al., 1999; Meunier and Bachevalier, 2002) except for the exclusion of activities that were either rare (viz., frown) or ambiguous (viz., piloerection, which can occur with both fear and aggression). Like earlier group $\mathrm{Rh}$, groups $\mathrm{ERh}$ and $\mathrm{PRh}$ did not differ significantly from controls in their general behavior recorded before and after stimulus presentation. In particular, none of the animals in these groups displayed the excessive manual and oral exploration of the testing apparatus often seen after lesions involving the amygdala (Meunier et al., 1999), nor did they engage in the excessive stereotypies of case Rh-5 (Meunier and Bachevalier, 2002). The results below therefore focus on responses to the stimuli, describing lesion-induced modifications relative to normal hallmark responses identified previously (Meunier et al., 1999; Meunier and Bachevalier, 2002), namely, (1) aggression and affiliation occurred only with the two "social" stimuli, with aggression equally triggered by the human and the monkey stimuli, whereas affiliation occurred mainly with the human, (2) defense varied across the 12 testing sessions, being lower during the first human presentation, the third monkey and snake presentations, and all three presentations of the rewarded object ("lower threat" sessions), than during the other six presentations ("higherthreat" sessions), (3) approach (viz., touch) and displacement behaviors (viz., environment exploration) characterized reaction to the rewarded object.

\section{Results \\ Entorhinal and perirhinal lesions yield undistinguishable changes}

A first set of analyses comparing groups ERh and PRh revealed no significant difference. The two groups were then compared with controls.

\section{Aggression}

The amount of aggressive responses to the social stimuli (human and monkey) was not significantly affected by either lesion (Fig. 1). However, the antagonistic postures (lunges) or gestures (mouth threats) typically seen in controls were observed in only three of the eight operated monkeys (ERh 1-2 and PRh-2) and were generally expressed in a tentative, low-key manner.

\section{Affiliation}

Affiliative responses to the human were significantly diminished in both groups ERh and PRh $\left(F_{(2,11)}=4.6 ; p=0.04\right.$; Dunnett's test, $p=0.02$ and $p=0.04$, respectively) (Fig. 1). All six controls displayed lip-smacks, grimaces, or both to the human, whereas only three operated monkeys (ERh 3-4 and PRh-2) showed some lip-smacks, again expressed in a tentative way.

\section{Defense}

Groups ERh and PRh did not reliably differ from controls during the six higher-threat sessions, but displayed a significant defense enhancement during the six lower-threat sessions $\left(F_{(2,11)}=4.3\right.$; $p=0.04$; Dunnett's tests, both $p$ values $=0.03)($ Fig. 1$)$. Operated monkeys' failure to diminish their defense with lower threat was confirmed by a three by two ANOVA revealing a significant group by threat interaction $\left(F_{(2,11)}=3.9 ; p=0.05\right)$.

\section{Other behaviors}

Approach was unchanged by ERh and PRh lesions. As in controls, oral exploration was rare and manual exploration was limited to displacing the object to retrieve the food reward hidden under- 
neath. Also, none of the monkeys with separate lesions presented the abnormal motor stereotypies seen in case Rh-5.

Both separate lesions replicate the effects of combined rhinal lesions Direct comparisons of groups ERh, PRh, and Rh yielded no significant difference. All the changes or lack thereof reported above, relative to controls, after separate lesions, replicate those following combined lesions (Fig. 1), namely, like groups ERh and PRh, group Rh displayed (1) slightly reduced, and often toned-down, antagonistic reactions to the two social stimuli, (2) a significant reduction of affiliative responses to the human stimulus (Dunnet's test for Rh, $p=0.04$ ), and (3) a defense enhancement that was most salient during the six lower-threat sessions (Dunnet's test for Rh, $p=0.05$ ).

No correlation was found between the animal's affective scores and the volume of entorhinal or perirhinal damage. Even the most selective entorhinal lesions with minimal extra-damage to posterior perirhinal and temporal polar areas (ERh 1 and 3 ) (see Fig. 3) yielded notable defense increases.

\section{Rhinal damage suppresses some, but not all, memory-dependent modulations of defense}

Failure to adapt to lower threat could reflect an inability to modulate behavior based on previous experience. To test this hypothesis, we carefully examined all possible memory-dependent variations of defense scores: across stimuli, over weeks for each stimulus, and across reward conditions for the object. For these analyses, the three experimental groups were pooled, because they did not differ from each other, to reduce the risk of type II error (missing a significance difference) associated with small samples.

\section{Remote memory influence and stimuli differentiation}

Knowledge acquired before the experiment likely guides controls' different responses to the four stimuli (Fig. 2A). The snake, a predator-like item, elicited more fear than the unknown social stimuli (snake vs mean for human and monkey over 3 weeks: paired $t$ test, $t=8.2 ; p<0.001$ ), which in turn were more threatening than the object, the most familiar item because of previous DNMS testing (social stimuli vs rewarded object over 3 weeks: $t=-2.9 ; p=0.03$ ). The speciestypical negative bias against the snake, presumably acquired early in life (Mineka et al., 1980), was spared in operated animals (snake vs mean for social stimuli over 3 weeks: paired $t$ test, $t=$ $2.4 ; p=0.04)$. In contrast, the two other classes of stimuli elicited the same exaggerated amount of defense ( $\mathrm{N}$ vs all $\mathrm{Rh}$ : social stim-
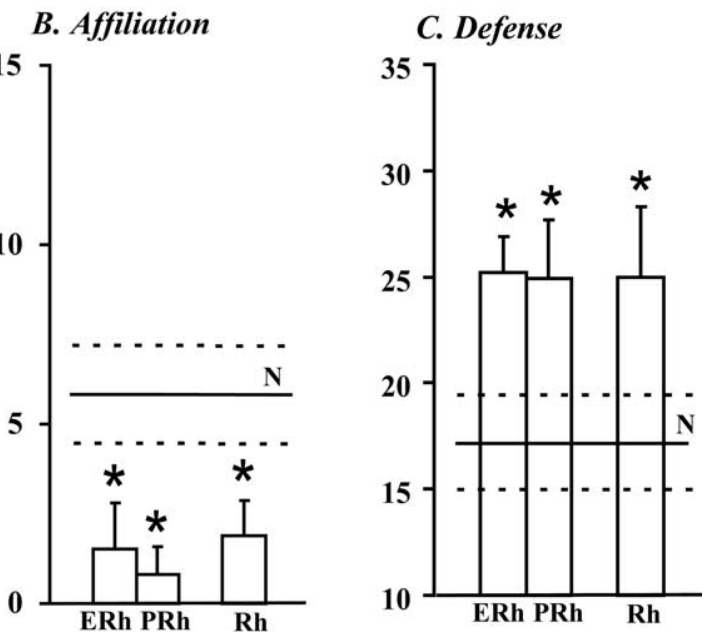

Figure 1. Separate entorhinal (ERh) or perirhinal (PRh) lesions both replicate the effects of combined (Rh) lesions. Relative to

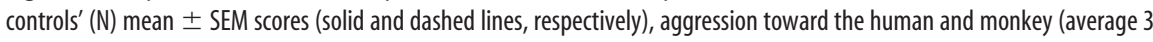
weeks) tends to be attenuated $(\boldsymbol{A})$, affiliative gestures to the human are significantly reduced (average 3 weeks) $(\boldsymbol{B})$, and defense during lower-threat sessions (average 6 sessions) is significantly increased $(\boldsymbol{C}) .^{*} p<0.05$ (Dunnett test). Scores are the cumulated duration of the activities composing each category and can thus exceed the 20 s length of stimulus presentation.

\section{A. Stimuli}

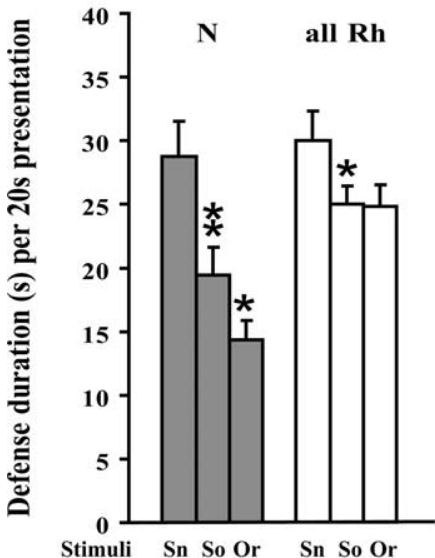

\section{B. Weeks}

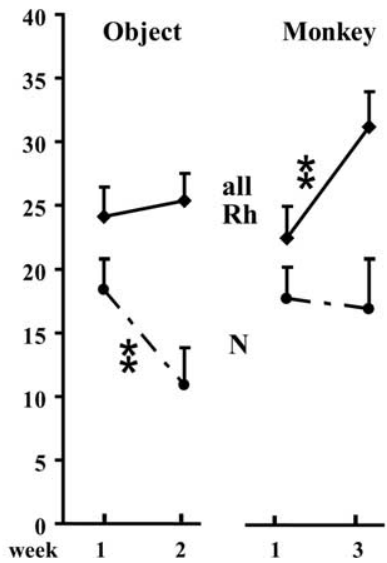

C. Reward

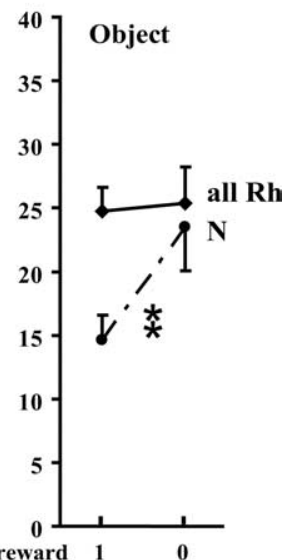

Figure 2. Memory-dependent modulations of defense in monkeys with either separate or combined entorhinal and perirhinal ablations (all Rh, $n=13$ ) and controls $(\mathrm{N}, n=6)$. $\boldsymbol{A}$, Remote memory (before the experiment) and stimulus identification. Controls (gray bars) fear the snake (Sn) more than the social (human and monkey) stimuli (So), and the social stimuli more than the rewarded object (0r). The former down-modulation is evident, albeit less marked, in operated monkeys, but not the latter (white bars). Each bar is the average ( \pm SEM) over three (Sn), six (So), or nine (Or) 20 s presentations. $\boldsymbol{B}$, Long-term memory (throughout the 3 weeks). Controls show a sharp defense drop on the second week of rewarded object presentations relative to the first week, that is absent in operated monkeys. Conversely, operated monkeys show a sharp defense rise on the third weekly monkey presentation relative to the first one that is lacking in controls. Each weekly score corresponds to one (monkey) or three (rewarded object) 20 s presentations ( \pm SEM).C, Short-term memory (within a daily session) and reward omission effect. Over the first 2 weeks of testing, omission of the reward on the fourth daily presentation of the object relative to the immediately preceding three rewarded presentations elicits a defense increase only in controls. Scores are averaged over six (reward $=1$ ) or two (reward $=0$ ) 20 s presentations. ${ }^{*} p<0.05 ;{ }^{* *} p<0.01$.

uli, $t=-2.2, p=0.046$; rewarded object, $t=-4.0, p=0.001$ ). However, operated monkeys did somewhat differentiate them because they showed move-away activities toward social stimuli, whereas they presented only eye aversion toward the less threatening object.

Long-term memory influence across weeks

In controls, changes across weekly encounters with the stimuli took the form of a clear-cut defense drop on the second, relative to the first object presentation (paired $t$ test on means over three rewarded presentations: $t=4.6 ; p=0.006$ ) (Fig. $2 B$ ). This down- 


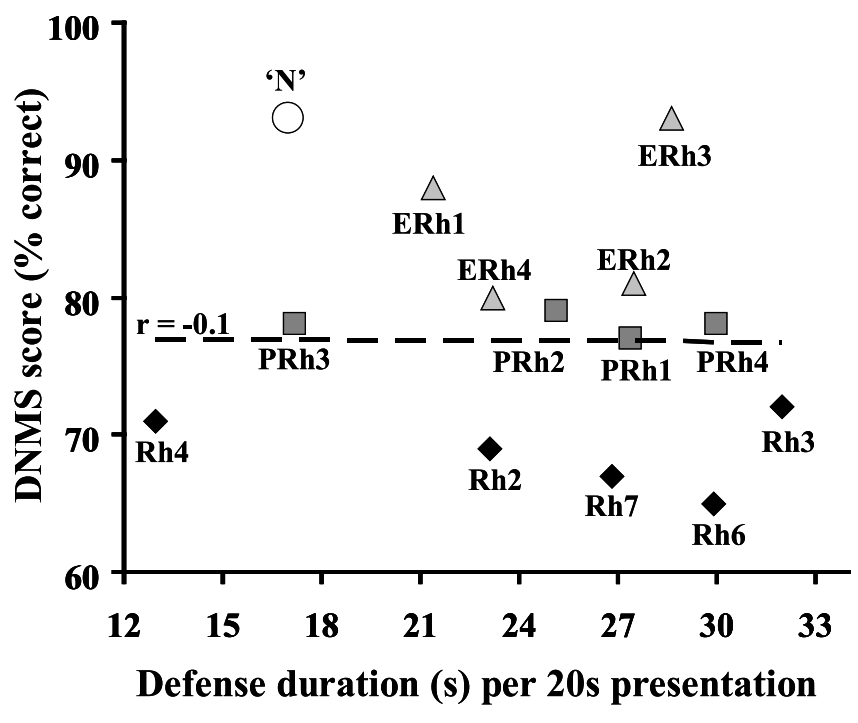

Figure 3. Lack of correlation between affective and recognition memory scores in monkeys with rhinal damage. Mean scores over the six extended delays and list lengths of DNMS (Meunier et al., 1993) are reported on the $y$-axis (the lower the score, the greater the deficit). Mean defense scores (duration) during lower-threat situations are reported on the $x$-axis. For comparison, the large open dot (' $\mathrm{N}$ ') depicts the mean DNMS score of the four controls in the memory study and the mean defense score of the six (different) controls in the present study. For monkeys with rhinal damage, labels correspond to case numbers used throughout studies, and $r$ is the Pearson's correlation coefficient for the 13 cases.

modulation was absent in monkeys with rhinal damage, which maintained the same overly defensive behavior toward the object across weeks (two by two ANOVA, week 1 vs 2: group, $F_{(1,16)}=$ 5.7, $p=0.03$; week, $F_{(1,16)}=1.7, p=$ not significant; group by week, $\left.F_{(1,16)}=5.1, p=0.04\right)$. Yet, operated monkeys did modify their defense behavior over the 3 weeks of the experiment. This took the form of a defense rise on the third, relative to the first monkey presentation (paired $t$ test: $t=-3.9 ; p=0.002$ ), which did not occur in controls (two by two ANOVA, week 1 vs 3: group, $F_{(1,17)}=5.6, p=0.03$; week, $F_{(1,17)}=4.35, p=0.05$; group by week, $\left.F_{(1,17)}=6.3, p=0.02\right)$.

Short-term memory influence and reward omission

Over both weeks 1 and 2, the unexpected omission of the reward during the fourth daily object presentation triggered a clear-cut defense increase in controls relative to the three preceding rewarded presentations (paired $t$ test: $t=-3.6 ; p=0.02$ ) (Fig. $2 C$ ). Operated animals again failed to display such a regulation of defense responses toward the object (two by two ANOVA: group, $F_{(1,16)}=2.8, p=0.11$; reward, $F_{(1,16)}=8.6, p=0.01$; group by reward, $\left.F_{(1,16)}=6.4, p=0.02\right)$.

\section{Emotional changes are not correlated with DNMS performance in rhinal groups}

To further explore possible links between the affective impact of rhinal lesions and their mnemonic consequences, we performed Pearson correlation analyses across all three groups (ERh, PRh, and $\mathrm{Rh}$ ) between each affiliative, aggressive, defense, and approach measure and the animals' own recognition memory performance (Meunier et al., 1993). No significant correlation was found between any category or activity score and memory performance, regardless of the DNMS score considered [overall performance (average over six extended delays and list lengths of objects as displayed in Fig. 3), score for the longer delay (120 s), or the longer list length (10 objects)]. Greater memory deficits failed to predict greater defensiveness. Instead, the one monkey with virtually normal DNMS performance (ERh 3) was as exaggeratedly defensive as the one with the poorest DNMS score (Rh 6). Conversely, monkeys with identical (PRh 3 and 4) or nearly identical (Rh 3 and 4) DNMS scores nevertheless fell at opposite ends of the defense spectrum.

\section{Discussion}

Rhinal lesions reliably disrupt monkeys' affective responses Large MTL removals (Bachevalier and Meunier, 2005) or amygdala aspiration or neurotoxic lesions (Meunier et al., 1999) in adult monkeys generally lead to drastic behavioral abnormalities that any casual observation by an untrained eye can detect. In contrast, rhinal damage results in subtle changes that can easily go unnoticed, even in the course of the daily testing entailed by memory experiments. Ethoexperimental quantitative evaluation of the behavior of five monkeys with rhinal ablations provided preliminary evidence that these changes are nevertheless significant (Meunier and Bachevalier, 2002). The first benefit of the present study is to replicate this original finding in eight new monkeys. This larger sample increases confidence that lesions limited to rhinal areas (i.e., devoid of direct damage to the overlying amygdala) reliably alter behavioral responses to affectively salient stimuli.

\section{Affective changes after rhinal damage do not resemble Klüver-Bucy symptoms}

The present findings also confirm the distinct socioemotional consequences of rhinal and amygdala damage. In the same behavioral paradigm, most monkeys with lesions involving the amygdala displayed typical Klüver-Bucy signs, that is, a drastic reduction of fear associated with excessive affiliation toward the social stimuli, and compulsive manual and oral exploration of nonsocial items, whether positive or negative (Meunier et al., 1999; Bachevalier and Meunier, 2005). None of these symptoms was observed in any of the monkeys with separate or complete rhinal ablations. The majority displayed, on the opposite, decreased affiliation and enhanced defense. Thus, although rhinal areas, in particular their rostral portions (Saunders and Rosene 1988), are strongly interconnected with the amygdala, the contributions of the two structures to affective functions likely differ.

Separate entorhinal and perirhinal ablations yield similar socioemotional changes despite their markedly different effects on visual recognition memory

The main strength of the present study is to provide direct comparison, in the same animals, of the affective and cognitive impacts of rhinal damage. Perirhinal damage was mostly responsible for the DNMS deficit after complete rhinal ablations (Meunier et al., 1993). In contrast, either of the separate lesions was sufficient to produce the affective changes after complete rhinal ablations. The monkeys' affiliative or defense scores were totally unrelated to their own DNMS scores. Most notably, the same exaggerated defense was observed in one entorhinal case with virtually normal DNMS performance and in the rhinal case that presented the most severe DNMS deficit. Therefore, the affective changes after rhinal damage clearly do not result from the disruption of the type of memory processes taxed by DNMS, that is, short-term novelty/familiarity judgments of socioemotionally meaningless objects.

\section{Memory- versus anxiety-centered interpretations}

Current models associate rhinal cortex not only with recognition memory as measured by DNMS, but also with other memory 
processes including long-term familiarization, associative memory, and perceptual identification of complex visual stimuli (Murray et al., 2005 and companion papers). It therefore remains possible that the affective changes described here derive from disruption of one of these processes for which both rhinal areas would be equally important. Alternatively, however, these changes may reflect a direct contribution of rhinal areas to the normal regulation of socioemotional responses. Monkeys with rhinal damage presented an intriguing pattern of behavior which combined an inability to reduce defense in the presence of the positive object (even in presence of a reward or after repeated presentations) with some spared abilities to increase defense in presence of negative stimuli (witnessed by their intact speciestypical snake fear and their abnormal sensitization over repeated presentations of the monkey stimulus, which was the only truly threatening stimuli because it was abruptly thrust toward the animal at midpresentation). Monkeys with rhinal damage, thus, did not systematically fail to recognize stimuli value and potential danger. Rather, they behaved as if they systematically over-rated the risk inherent in the stimuli. Such a negatively biased evaluation of affective stimuli is not predicted by current mnemonic/ perceptual models of the hippocampal-rhinal duo. It is more reminiscent of Gray and McNaughton's (2000) alternative model of the hippocampus as a substrate for conflict resolutions between approach and avoidance. In Gray and McNaughton's model, the hippocampus inhibits approach in case of conflict by increasing the weight of affectively negative information. Rhinal areas functions are not clearly specified. Based on the present findings, these areas could modulate the interactions between the hippocampus and the amygdala to reduce anxiety in presence of familiar positive stimuli.

\section{Rhinal cortex as a site for cognitive, memory-based, modulation of affective responses?}

Too few studies questioning specifically the role of rhinal areas in affective functions have been performed in primates, human and nonhuman, to determine which of the two explanations proposed above, the memory- or the anxiety-centered views, best accounts for the rhinal damage effects described here. We believe that the two views are not mutually exclusive and that our understanding of rhinal functions might be improved by the introduction of an affective dimension to current cognitive models. A recent functional magnetic resonance imaging study showing that the hippocampus and entorhinal cortex both contribute, in complementary but distinct ways, to anxiety-related modulations of pain perception (Ploghaus et al., 2001) strongly encourages further exploration of this issue in primates. Anatomically, the rhinal areas represent the end point and first polysensory station of the ventral visual pathway, a gate to and from the hippocampus, and also an integral part of the amygdalo-orbital network believed to be important for socioemotional behaviors (Suzuki, 1996). They are, thus, ideally placed to integrate the perceptual, mnemonic, and affective signals that are all mandatory for appropriate cognitive appraisal of the affective meaning of sensory stimuli.

\section{References}

Amaral DG (1992) Anatomical organization of the primate amygdaloid complex. In: The amygdala: neurobiological aspects of emotion, memory, and mental dysfunction (Aggleton JP, ed), pp 1-66. New York: Wiley.

Bachevalier J, Meunier M (2005) Neurobiology of social-emotional cognition in nonhuman primates. In: The cognitive neuroscience of social behaviour (Easton A, Emery N, eds), pp 19-58. Psychology.

Gray JA, McNaughton N (2000) The neuropsychology of anxiety, Ed 2. Oxford, UK: Oxford UP.

Klüver H, Bucy P (1939) Preliminary analysis of functioning of the temporal lobes in monkeys. Arch Neurol Psychiat 42:979-1000.

Meunier M, Bachevalier J (2002) Comparison of emotional responses in monkeys with rhinal cortex and amygdala lesions. Emotion 2:147-161.

Meunier M, Murray EA, Bachevalier J, Mishkin M (1993) Effects on visual recognition of combined and separate ablations of the entorhinal and perirhinal cortex in rhesus monkeys. J Neurosci 13:5418-5432.

Meunier M, Hadfield W, Bachevalier J, Murray EA (1996) Effects of rhinal cortex lesions combined with hippocampectomy on visual recognition memory in rhesus monkey. J Neurophysiol 75:1190-1205.

Meunier M, Bachevalier J, Murray EA, Málková L, Mishkin M (1999) Effects of aspiration vs neurotoxic lesions of the amygdala on emotional responses in monkeys. Eur. J Neurosci 11:4403-4418.

Meunier M, Alvarado MC, Bachevalier J (2001) Emotional responses in monkeys with entorhinal or perirhinal cortex lesions. Soc Neurosci Abstr 27:455.2.

Mineka S, Keir R, Price V (1980) Fear of snakes in wild- and lab-reared rhesus monkeys. Anim Learn Behav 8:653-663.

Murray EA, Graham KS, Gaffan D (2005) Perirhinal cortex and its neighbours in the medial temporal lobe: contributions to memory and perception. Q J Exp Psychol B 58:378-396.

Otto T, Cousens G, Herzog C (2000) Behavioral and neuropsychological foundations of olfactory fear conditioning. Behav Brain Res 110:119-128.

Ploghaus A, Narain C, Beckmann CF, Clare S, Bantick S, Wise R, Matthews PM, Nicholas J, Rawlins P, Tracey I (2001) Exacerbation of pain by anxiety is associated with activity in a hippocampal network. J Neurosci 21:9896-9903.

Saunders RC, Rosene DL (1988) A comparison of the efferents of the amygdala and the hippocampal formation in the rhesus monkey: I. Convergence in the entorhinal, prorhinal, and perirhinal cortices. J Comp Neurol 271:153-184.

Scoville WB, Milner B (1957) Loss of recent memory after bilateral hippocampal lesions. J Neurol Neurosurg Psychiat 20:11-21.

Stefanacci L, Clark RC, Zola SM (2003) Selective neurotoxic amygdala lesions in monkeys disrupt reactivity to food and object stimuli and have limited effects on memory. Behav Neurosci 117:1029-1043.

Suzuki WA (1996) The anatomy, physiology and functions of the perirhinal cortex. Curr Opin Neurobiol 6:179-186 